\title{
Automatic differentiation: a tool for variational data assimilation and adjoint sensitivity analysis for flood modeling
}

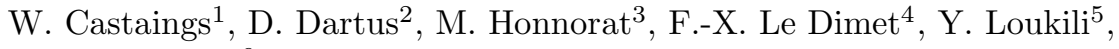 \\ and J. Monnier ${ }^{6}$ \\ 1 INRIA/IDOPT william. castaings@imag. $f r^{*}$ \\ ${ }^{2}$ INPT - IMFT/HYDRE dartus@imft.fr \\ 3 INPG - INRIA/IDOPT marc.honnorat@imag.fr ${ }^{\star \star}$ \\ 4 UJF - INRIA/IDOPT francois-xavier.le-dimet@imag.fr \\ ${ }^{5}$ INRIA/IDOPT youssef.loukili@imag. $f r^{\star \star \star}$ \\ ${ }^{6}$ INPG - INRIA/IDOPT jerome.monnier@imag.fr
}

\section{Introduction}

Flooding is the result of complex interactions between the components of water cycle and the forecast of such catastrophic events requires a completely integrated approach (models and data) for the hydro-meteorological prediction chain. The modeling of flood generation and propagation involves catchment scale hydrology and river hydraulics. Actually, every model component only leads to an approximation of the geophysical reality, since the underlying physics formulation and the model inputs are all sources of uncertainty. Understanding, analysis and reduction of this uncertainty induce the following issues:

- the sensitivity analysis is a key issue for providing physical insight into the model dynamics.

- the initial and boundary conditions which are essential to mathematical closure and drive the considered system, remain very difficult to estimate.

- the empirical parameters associated with model formulation must be calibrated or identified.

- the estimation of the systematic model error (correction term) could be an additional prediction value.

A deterministic approach dealing with the aforementioned estimation and sensitivity analysis problems results in the need of computing the derivatives

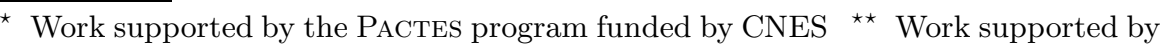
a CNRS doctoral grant (BDI) co-funded by CNES. ${ }^{\star \star \star}$ Post-doctoral work funded by Région Rhône-Alpes ("Prévention numérique de crues" project).
} 
of a function of model output variables with respect to input variables. Modern automatic differentiation (AD) tools such as TAPENADE $[14,9,8]$ provide an easier and safe way to fulfill this need.

Two applications are presented in this paper: variational data assimilation $[5,1]$ and adjoint sensitivity analysis $[2,3]$.

\section{The Adjoint Method}

The evolution of the state of many time-dependent physical systems can be described by a system of differential equations. For a given model, the value of the state variable $y$ is driven by the control variables which are potentially all model inputs. For the general presentation of the adjoint method, we will consider, as control variables, the initial condition $u$ and a model parameter $v$ :

$$
\left\{\begin{array}{l}
\left.\left.\frac{\partial y}{\partial t}(t)+A(y(t), v)=0 \quad \forall t \in\right] 0, T\right] \\
y(0)=u
\end{array}\right.
$$

where $A$ is a partial differential operator which may be non-linear. Let $\phi$ be a general objective function which depends on the control variables through the state variable :

$$
\phi(u, v)=\int_{0}^{T} \varphi(y(u, v ; t)) d t,
$$

where $\varphi$ is a sufficiently smooth functional. The adjoint method makes it possible to efficiently compute the partial derivatives of a function of the model state variable with respect to control variables [10]. If $p$ is defined as the solution of the adjoint model

$$
\left\{\begin{array}{l}
\frac{\partial p}{\partial t}(t)-\left[\frac{\partial A}{\partial y}(y(t), v)\right]^{*} \cdot p(t)=\frac{\partial \varphi}{\partial y}(t) \quad \forall t \in[0, T[, \\
p(T)=0
\end{array},\right.
$$

then we obtain a simple expression of the partial derivatives of the functional:

$$
\frac{\partial \phi}{\partial u}(u, v)=-p(0) \quad \text { and } \quad \frac{\partial \phi}{\partial v}(u, v)=\int_{0}^{T}\left[\frac{\partial A}{\partial v}(y(t), v)\right]^{*} \cdot p(t) d t .
$$

All partial derivatives are calculated with a single forward integration of the direct model (1) followed by a single backward integration of the adjoint model (3). Another advantage of this method lies in the fact that the homogeneous part of the adjoint equations is independent of the functional $\varphi$. In other words, the same model can be used to calculate the derivatives of several functionals without major modifications.

The calculation of partial derivatives of a functional is useful in several domains of flood simulation. Here, we are especially interested in two essential applications: variational data assimilation and adjoint sensitivity analysis. 
Variational data assimilation consists in finding the control variables that minimize a cost function measuring the discrepancy between the state variable of the model and data obtained from the observation of the physical system. An efficient minimization of the cost function is carried out by using a descent algorithm requiring the computation of its gradient. The adjoint sensitivity analysis consists in determining the contribution of all model inputs to the variation of a response function. Instead of performing finite difference approximation of the gradient, requiring extensive direct model computations (brute force method), a single run of the adjoint method provides all sensitivities. Various applications of the adjoint method were investigated for environmental problems in the framework of the IDOPT project $[12,13,16,17,18]$.

In practice, the numerical computation of the gradient of the functional $\phi$ is performed by an implementation of the adjoint method which requires the construction of an adjoint code. Since the best representation of the functional is the associated computer code, it is better to use the adjoint of the implementation of the direct model instead of the implementation of the continuous adjoint model to compute the exact value of the gradient. The use of the AD tool TAPENADE [14] makes it possible to save a lot of time in building the adjoint code and prevents from many human errors.

\section{River Hydraulics}

The forecast of floods requires an accurate modeling of river flows. The most commonly used mathematical models for operational purposes in river hydraulics rely on the Shallow Water equations (SWE). The two-dimensional SwE are derived from the three-dimensional Navier-Stokes equations by a vertical integration under the hydrostatic assumption. In the conservative formulation, the state variables are the water depth $h$ and the discharge $\mathbf{q}=h \mathbf{u}$ where $\mathbf{u}$ is the depth-averaged velocity vector. If we consider a computational domain $\Omega$ with a boundary $\Gamma$, the two-dimensional swe can be written as follows:

$$
\left\{\begin{aligned}
\partial_{t} h+\operatorname{div}(\mathbf{q})=0 & \text { in } \Omega \times] 0, T] \\
\partial_{t} \mathbf{q}+\operatorname{div}\left(\frac{1}{h} \mathbf{q} \otimes \mathbf{q}\right)+\frac{1}{2} g \nabla h^{2} & \\
+g h \nabla z_{b}+g \frac{n^{2} \mathbf{q}\|\mathbf{q}\|_{2}}{h^{7 / 3}}=0 & \text { in } \Omega \times] 0, T] \\
h(0)=h_{0}, \quad \mathbf{q}(0)=\mathbf{q}_{0} & \text { in } \Omega
\end{aligned}\right.
$$

where $g$ is the magnitude of the gravity, $z_{b}$ the bed elevation, $n$ the Manning roughness coefficient, $h_{0}$ and $\mathbf{q}_{0}$ the initial conditions. Moreover, we must add boundary conditions. For an inlet $\Gamma_{\mathrm{in}}$, a discharge $\mathbf{q}_{\text {in }}$ is imposed:

$$
\left.\left.\mathbf{q}_{\mid \Gamma_{\mathrm{in}}}(t)=\mathbf{q}_{\text {in }}(t) \quad \forall t \in\right] 0, T\right] .
$$


Concerning an outlet $\Gamma_{\text {out }}$, we can either prescribe a water depth $h_{\text {out }}$ or impose Neumann conditions:

$$
\begin{array}{lll}
h_{\left.\right|_{\Gamma_{\text {out }}}}(t)=h_{\text {out }}(t),\left.\quad \frac{\partial \mathbf{u}}{\partial \mathbf{n}}\right|_{\Gamma_{\text {out }}}(t)=0 & \forall t \in] 0, T] \\
\text { or }\left.\quad \frac{\partial h}{\partial \mathbf{n}}\right|_{\Gamma_{\text {out }}}(t)=0,\left.\frac{\partial \mathbf{q}}{\partial \mathbf{n}}\right|_{\Gamma_{\text {out }}}(t)=0 & \forall t \in] 0, T]
\end{array}
$$

The state of the flow is determined by the initial condition (Cauchy problem) but also by the model parameters $\left(z_{b}\right.$ and $\left.n\right)$ and the boundary conditions. Actually, in order to carry out a simulation of a real flow, it is necessary to have a good knowledge of these model inputs. However, they are incompletely known in practice, and when an approximation is available, it is often subject to uncertainty. For example, the flood plain elevation is measured using remote sensing techniques (photogrammetry, LIDAR) and bed elevation data is made up of ground surveyed cross sections. The collected data consist in a set of scattered points used to assign an elevation value to each computational grid point using interpolation techniques. Unfortunately, the raw data is usually approximate, incomplete or sometimes simply missing. Moreover, the interpolation induces additional numerical approximation. Other model inputs cannot even be directly measured and should be defined thanks to the modelers' expertise. For example, the estimation of roughness is generally based on land use classifications and empirical tables where a roughness coefficient is assigned to each land cover type. Besides, a model is never perfect since it cannot take into account all the physics of the system, and its implementation induces numerical approximations. Therefore, a simulation can never reflect exactly the physical reality. However, it is possible to represent some parts of the model errors by an additional term in the equations [16], introducing new control variables.

Furthermore, some observations of the flow state may be available, such as water depth, water level or velocity measurements. These should be in accordance with the simulation results. Therefore, the problem to be addressed consists in identifying a set of control variables consistent with both the simulation results and the hydraulic reality represented by observation data. Hence,

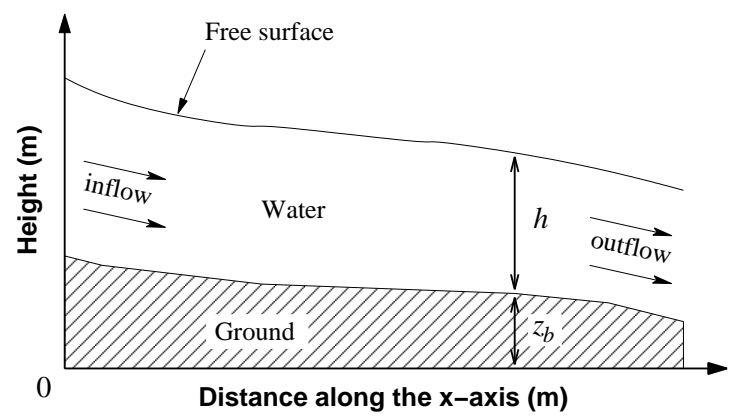

Fig. 1. One-dimensional vertical cut of the computational domain 
we use variational data assimilation for the identification of initial and boundary conditions, model parameters, bed elevation and for the evaluation of a systematic model error. This method consists in minimizing a cost function measuring the discrepancy between the state of the simulated flow and the available observations of the real flow. The minimization is performed by a limited memory quasi-Newton algorithm [7] which requires the computation of the partial derivatives of the cost function with respect to the control variables of the model. The derivatives are computed by the adjoint method requiring the use of an adjoint code.

The swe (4) are solved using the finite volume method and the HLLC approximate Riemann solver $[15,11]$. The direct program is written in Fortran 90 and is made up of about 1600 lines of code. The adjoint code was obtained thanks to the AD tool TAPENADE [8]. The raw code produced by TAPENADE had to be manually modified to make it work properly. The adjoint of some non-differentiable statements was rewritten and some unnecessary storage of the state variable was removed. Before this optimization, the adjoint could not run on a simple test case because the needed amount of memory was too large (more than 2 gigabytes). After this optimization, the memory footprint is only 16 megabytes and the ratio between the execution time of the adjoint code and that of the direct code is about 3.5.

Two numerical experiments of data assimilation are presented. They actually consist of twin experiments, where observation data are computed by the direct model with a set of known parameters. Then, a perturbation is applied to the latter, modifying the simulation results. Afterwards, the variational data assimilation method is used to retrieve the original value of the parameters. The same adjoint code is used in both experiments.

The first one concerns the identification of the bed elevation $z_{b}$ in a rectangular channel. The channel is $20.5 \times 2$ meters, the reference bed elevation is defined by $z_{b}(x, y)=0.2-\frac{1}{20}(x-10)^{2}$ if $x \in[8 ; 10]$ and $z_{b}(x, y)=0$ otherwise for all $y \in[0 ; 2]$. A constant discharge $\mathbf{q}_{\text {in }}$ of $2 \mathrm{~m}^{3} \mathrm{~s}^{-1}$ is imposed at the inlet and a constant water depth $h_{\text {out }}$ of 0.6 meters is prescribed at the outlet. This configuration leads to a steady flow featuring an hydraulic jump after the bump. A vertical cut of the computational domain is plotted in Fig. 2. The observations of the water depth $h^{\text {obs }}$ and the velocity $\mathbf{u}^{\text {obs }}$ are created from

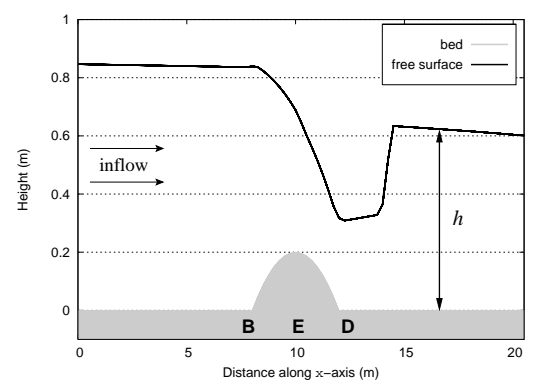

Fig. 2. Rectangular channel with a bump: vertical cut of the computational domain 
this reference configuration: they are defined as equal to the state variables of the steady flow for each computational point. It should be noted that in this case, the observation data is time-independent. However, nothing prevents us from using observations varying in time.

A simulation is carried out with a modified configuration over a period of $T=3$ seconds: a flat bed defined by $z_{b} \equiv 0$ is used with the reference steady state as an initial condition. As a result, the water flow is disrupted and becomes unsteady. In order to use the variational data assimilation method to retrieve the reference bed elevation, we introduce the following cost function:

$$
j_{1}\left(z_{b}\right)=\frac{1}{2} \int_{0}^{T}\left(\left\|h(t)-h^{\mathrm{obs}}(t)\right\|_{\Omega}^{2}+\left\|\mathbf{u}(t)-\mathbf{u}^{\mathrm{obs}}(t)\right\|_{\Omega}^{2}\right) d t .
$$

The gradient of this cost function is computed with the adjoint code and is used as an input for the minimization algorithm. In Fig. 3 (a), the cost function and the norm of its gradient, both normalized by their initial value, are plotted against the number of iterations of the minimization process. Figure 3 (b) shows the bed elevation for several steps of the minimization process. We

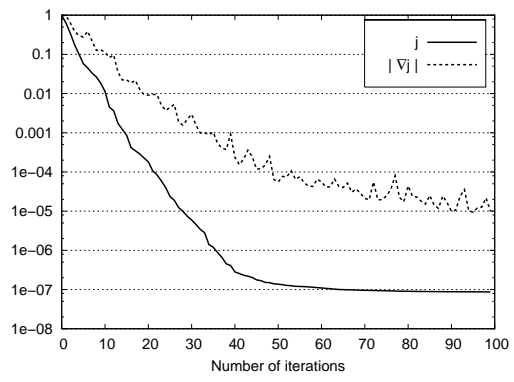

(a) Convergence of the cost function

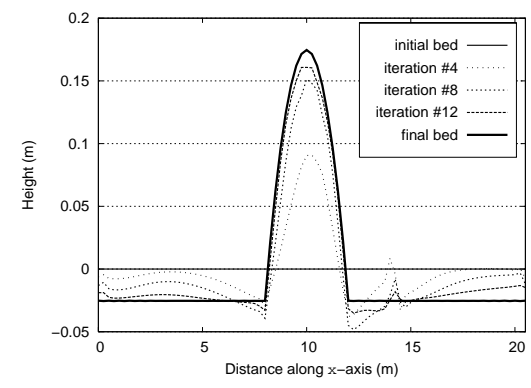

(b) Convergence of the bed elevation

Fig. 3. Rectangular channel with a bump: convergence of the minimization process

can see that convergence has been achieved and the original bump on the bed is retrieved. However, even if the shape of the bump is correctly identified, one can notice a constant offset between the retrieved bed elevation and the original one. This can be explained by the fact that information on the actual water level is available neither in the formulation of the cost function nor in the observations. There is only information on the water depth which is the difference between the water level and the bed elevation. Therefore, the latter can be identified only up to a constant bias.

The second experiment concerns the identification of the upstream boundary condition $\mathbf{q}_{\text {in }}$ during a flood event. We consider a 200 meters long rectangular channel with a constant slope of $0.5 \%$. The initial conditions consist in a steady flow initiated by the prescription of a constant discharge of $10 \mathrm{~m}^{3} \mathrm{~s}^{-1}$ at the inlet. Flooding is created by the modification of the upstream boundary condition: for a period of $T=80$ seconds, it is defined by 
$\mathbf{q}_{\text {in }}(t)=10+5 t \exp \left(-\frac{(t-5)^{2}}{100}\right)$. The corresponding hydrograph is plotted in Fig. 4 (a). One can see the propagation of the spate in Fig. 4 (b) where the water surface profile is displayed at several time steps. The water depth is

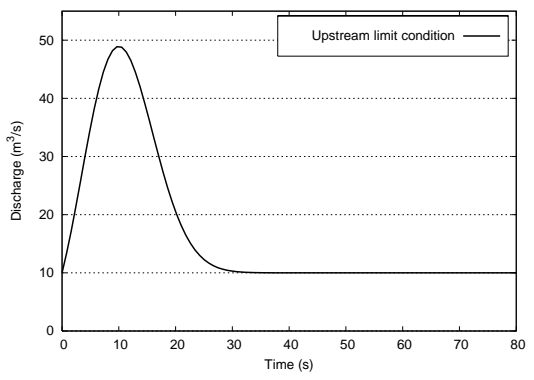

(a) Upstream boundary condition

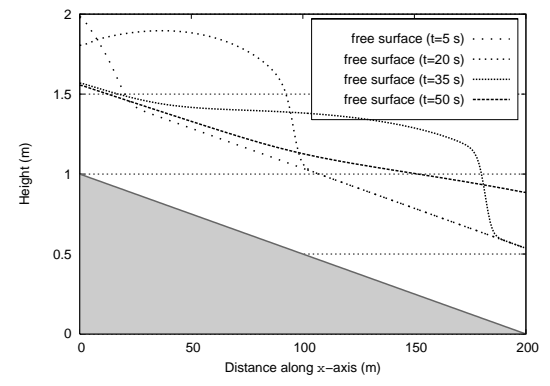

(b) Free surface evolution

Fig. 4. Rectangular channel with a constant slope: configuration

recorded continuously in time at a measurement point located at a given distance $x_{m}$ from the upstream boundary of the domain. This measurement is used as an observation $h^{\text {obs }}(t)$ of the water depth during the flooding event. It makes it possible to define a cost function measuring the discrepancy between the water depth $h$ and the observations $h^{\text {obs }}$ at the point $x_{m}$ :

$$
j_{2}\left(\mathbf{q}_{\text {in }}\right)=\frac{1}{2} \int_{0}^{T}\left|h\left(x_{m}, t\right)-h^{\mathrm{obs}}(t)\right|^{2} d t .
$$

From the initial hypothesis of a steady flow where the discharge is $10 \mathrm{~m}^{3} \mathrm{~s}^{-1}$, the experiment consists in identifying the hydrograph that is at the origin of the spate. For that purpose, we use the variational data assimilation method. If the point of measurement is situated near the inlet ( $x_{m} \leq 40$ meters), the identification of the boundary condition is very good. In Table $1, \varepsilon$, the relative $L^{2}$ error between the identified discharge and the reference discharge is given for several values of the distance $x_{m}$. We conclude that the efficiency of the identification decreases when the distance of the point of measurement to the inlet increases.

The potential of variational data assimilation applied to river hydraulics was illustrated through two experiments. However, the tests were limited to the case of twin experiments where uncertainty on the data and assumptions

Table 1. Relative $L^{2}$ error on the identified boundary condition for several positions of the water depth measurement

\begin{tabular}{|c||c|c|c|c|c|c|c||}
\hline$x_{m}$ & $1 \mathrm{~m}$ & $20 \mathrm{~m}$ & $40 \mathrm{~m}$ & $80 \mathrm{~m}$ & $120 \mathrm{~m}$ & $180 \mathrm{~m}$ & $195 \mathrm{~m}$ \\
\hline$\varepsilon$ & $0.45 \%$ & $0.50 \%$ & $0.53 \%$ & $2.16 \%$ & $5.21 \%$ & $8.95 \%$ & $10.1 \%$ \\
\hline
\end{tabular}


on the model inputs are perfectly controlled. Assimilation of real observation data should be further investigated. The identification of a systematic model error would be valuable for an operational use of an hydraulic model. Moreover, the assimilation of observations of a different nature, such as trajectories or flood marks could bring additional information for the identification of control variables. A big asset of the adjoint method is that the same adjoint code can be used for all problems. Only the few lines of the adjoint code that correspond to the computation of the cost function are to be modified, which is very easy with an $\mathrm{AD}$ tool.

\section{Catchment Hydrology}

Flash flood events are usually generated by heavy convective precipitation over a relatively small area, but catchment hydrology plays a major role in their occurrence. Thus, the transformation of rainfall into runoff is a critical component for flash flood analysis. Recently, distributed hydrological models became an attractive approach for the modeling of watershed hydrology. Nevertheless, limited knowledge of model inputs (initial and boundary conditions, parameters) and observations of the hydrological response make the underlying problems of calibration, sensitivity analysis and uncertainty analysis very challenging.

The underlying physics of MARINE, a model developed by Estupina et al. [6] is adapted to events for which infiltration excess dominates the generation of the flood. For the temporal and spatial scales of interest, rainfall abstraction by infiltration is evaluated using the Green Ampt model and the resulting surface runoff (hillslope flow) is transferred using the Kinematic wave approximation (KWA). Lastly, river flow is routed with the full Saint-Venant equations, $1 D$ or $2 D$ depending on the valley configuration. The coupling with the river hydraulics component will not be discussed below. The simplification of mass and momentum conservation equations representing overland flow (KWA) is given by

$$
\frac{\partial h}{\partial t}+\frac{s^{1 / 2}}{n} \frac{\partial h^{5 / 3}}{\partial x}=r-i
$$

where $h$ is the flow depth, $n$ the Manning roughness coefficient, $s$ the slope in the steepest direction, $r$ the rainfall rate and $i$ the infiltration rate. A preliminary analysis of digital elevation model is carried out in order to compute a single steepest descent flow direction from four available directions for each cell. Then, (10) is solved using a simple explicit Euler scheme on the hillslope represented by a cascade of planes. In order to ensure convergence of the numerical scheme, a threshold value $u_{m}$ on flow velocities was introduced. In the right hand side term of (10) which represents the excess rainfall, the infiltration rate $i(t)$ is evaluated using the following procedure: 


$$
\begin{array}{ll}
r<i & i=r \\
r \geq i & i=\frac{d I}{d t}=K\left(\frac{\psi \eta \theta}{I}+1\right),
\end{array}
$$

where $I$ is the cumulative infiltration, $K$ the hydraulic conductivity, $\psi$ the suction force, $\eta$ the porosity and $\theta$ the relative initial moisture deficit. Equation (11) is solved using an implicit Euler scheme, and the resulting fixed point equation using the Newton method.

MARINE just like most hydrological models is far from being fully comprehensive and really simplify the complex hydrologic reality. In fact, improving physical understanding would increase the number of parameters to be calibrated. Event and physically based models like MARINE are easier to set up, have lower computational cost and require less parameters to be calibrated. However, they have short term memory (antecedent conditions should be provided for initial soil moisture) and the prescription of consistent parameters associated with the model formulation is a challenging task. In fact, the model formulation is only a conceptualization of the catchment dynamics and model parameters account for uncertainty associated to model formulation, model structure, forcing conditions and calibration data. Therefore, effective values for model parameters cannot be obtained from direct measurements; they are calibrated to make model results fit available observation data. Therefore, the potential of the adjoint method described in Sect. 2 should be investigated. However, MARINE just like most mathematical representations of catchment hydrology is strongly non-linear and involve multiple thresholds or switches due to the intrinsic nature or related conceptualization of the physical processes (rainfall, infiltration regimes, maximum infiltration capacity, etc.). Since introducing smoothing functions may lead to important inconsistencies between direct and adjoint models, AD seem to be an efficient alternative to obtain the required derivatives (i.e. sub-gradients). Hence MARINE adjoint model was developed using TAPENADE. After some minor code modifications, extensive validation and optimization were carried out for the differentiated code in order to conduce adjoint sensitivity and variational data assimilation experiments.

The rainfall-runoff relation is a typical case where the dimension of the system response to be analyzed is small compared to the number of input parameters to be prescribed. In this case, the adjoint model is very efficient in computing the gradient of a response function w.r.t. all parameters (see Cacuci [4] for a recent theoretical basis). Obviously, the choice of the forecast aspect to be analyzed will affect the analysis. However, multi-criteria and multi-variable analysis can be carried out using the previously described framework. A single run of the adjoint model for each criteria or response function will yield to the local sensitivities for all parameters.

Since the objective targeted by MARINE is an accurate representation of the rising limb of the flood, only the global response of the watershed (outlet hydrograph) will be analyzed. For flash flood events, the runoff coefficient 
and the maximum discharge are probably the most relevant quantities to be estimated. Thus, let us define

$$
g_{1}=\frac{\int_{0}^{T} q(t) d t}{\int_{0}^{T} \int_{0}^{\Omega} r(t) d \Omega d t} \quad g_{2}=\frac{\max _{t \in[0, T]} q(t)}{q_{r e f}},
$$

where $q(t)$ is the outlet discharge and $q_{r e f}$ the maximum discharge obtained when all rainfall is transformed into runoff (no infiltration). Sensitivities to $g_{2}$ are only defined during the rising limb and vanish during recession. In fact, the maximum discharge $q_{\max }$ is calculated during the temporal integration and when $q(t)$ is greater than the current $q_{\max }$, temporal increments in sensitivities correspond to sensitivities of $q(t)$ to model parameters. In addition, from the previously mentioned quantities, we can define $g_{3}$, an adimensionalized and normalized synthesis of the hydrograph:

$$
g_{3}=\frac{g_{1}}{\sqrt{g_{1}^{2}+g_{2}^{2}}}+\frac{g_{2}}{\sqrt{g_{1}^{2}+g_{2}^{2}}} .
$$

The examination of the sensitivities will allow us to investigate their hydrological meaning and analyze model behavior.

The chosen watershed is a very small catchment area $\left(25 \mathrm{~km}^{2}\right)$ from the upper part of Thoré basin. Given the basin features, no river flow routing was considered and uniform land use and soil type is assumed in order to facilitate the analysis. Manning roughness coefficient $n$ and Green Ampt model parameters $(K, \psi$ and $\eta)$ are derived a priori from published tables using information on land use and soil type. Concerning the rainfall forcing, real radar data (hydram from Meteo France) was lumped over the area. Since the flow directions are computed before the model integration, accounting for the slope $s$ in the sensitivity analysis would lead to systematic underestimation of its influence. Therefore, an adjoint sensitivity analysis was carried out w.r.t. model parameters $K, \psi, \eta, \theta$ and $n$. Moreover, the response function can also be differentiated w.r.t. numerical or algorithmic artifacts like $u_{m}$ which do not appear neither in continuous or discretized model equations. However, ranking the sources of uncertainty (i.e. the sensitivity of model response to parameters) requires a normalization of the adjoint variables. The scaled sensitivities are defined as

$$
s_{k}=\frac{\partial g}{\partial \alpha_{k}} \cdot \frac{\alpha_{k}}{g},
$$

with $g$ the response function, $\alpha_{k}$ the model parameter and $s_{k}$ the normalized sensitivity. Since parameters are fully distributed, the $L_{2}$ norms were computed in order to rank parameters influence on model response. A summary of the obtained results is given in Table 2 . One can see that $u_{m}$ which was set to $1 \mathrm{~ms}^{-1}$ has a greater impact on $g_{2}$ and $g_{3}$ than the calibration parameters. Extensive experiments were performed and show that kinematic shocks 
occur and propagate in the flow accumulation regions (i.e. in the drainage network) and lead to spurious velocities. Confrontations of brute force and adjoint sensitivity analysis confirm the importance, localization and temporal evolution of this phenomenon. In fact, using KWA, the underlying mathemat-

Table 2. Contributions (in \%) of model parameters to the hydrological response

\begin{tabular}{||c||c|c|c|c||}
\hline & $\eta, \theta, S_{f}$ & $K$ & $n$ & $u_{m}$ \\
\hline \hline$g_{1}$ & 19.51 & 37.57 & 3.87 & $3.77 \mathrm{E}-07$ \\
\hline$g_{2}$ & 1.65 & 3.10 & 7.27 & 84.66 \\
\hline$g_{3}$ & 10.20 & 19.63 & 4.32 & 45.41 \\
\hline
\end{tabular}

ical model is still non-linear and the occurrence of shocks seem inescapable specially when the complex watershed topography is represented by a cascade of planes. Therefore, appropriate shock fitting methods should be used in order to ensure that the numerical approximation of the kinematic wave does not dominate the parameter calibration issue. On the other hand, the results obtained with response function $g_{1}$ show that effect of this threshold on the runoff coefficient can be neglected and that the partition of rainfall into runoff and infiltration is mainly driven by hydraulic conductivity $K$. Moreover, it was shown that the wetter the soil is, the shorter is the decay of $i$ to $K$ and the larger is the influence of parameter $K$. In addition, a detailed analysis of the spatial and temporal patterns of the obtained sensitivities really provide physical insight into the model dynamics. In fact, all the cells of the watershed are solicited for infiltration from both direct rainfall and excess rainfall coming from upstream in the basin (run-on). The latter seems to be critical since the spatial pattern of sensitivity to all Green Ampt infiltration parameters is driven by the drainage network. For example, one can see in Fig. 5 the correspondence between slopes and sensitivity to $K$. Moreover,

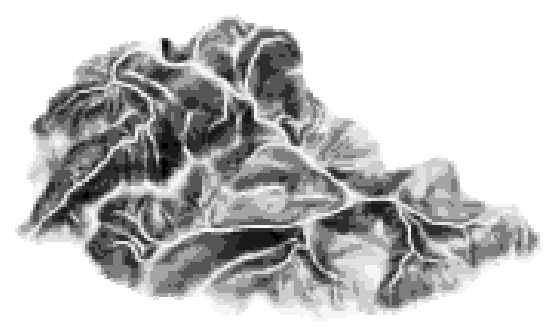

(a) DTM slopes

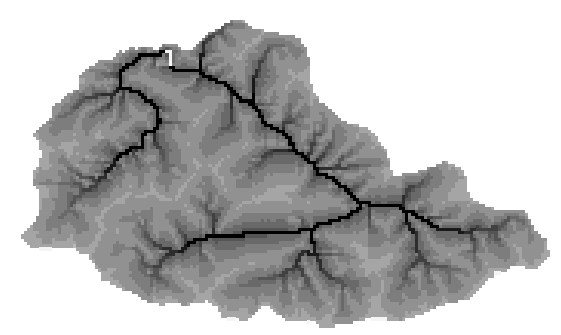

(b) Sensitivity to $K$

Fig. 5. Sensitivity to infiltration parameters and catchment slopes

Fig. 6 exhibits the temporal patterns of the sensitivities to model parameters. The thresholds related to rainfall dynamics and different infiltration regimes 
lead to similar thresholds on temporal increments of adjoint variables. One can see in Fig. 6 that the event was divided into four periods and once again the results are in agreement with the infiltration excess overland flow mechanism. In fact, during period 1, rainfall totally infiltrates to the unsaturated zone without intervention of model parameters. Then, once rainfall intensity becomes important (beginning of period 2) the infiltration from direct rainfall is immediate and run-on develops. This can be noticed by the rising of the sensitivity to $n$. During period 2, rainfall duration and intensity remain limited and do not produce rising of the hydrograph. On the contrary during period 3, rainfall is so intense and its duration so important that a large amount of runoff is produced. The runoff coefficient and associated statistical moments were computed (mean and variance) and showed thats during this period the runoff coefficient is very close to unity on the whole watershed. Therefore, the global influence of $K$ remains constant and the sensitivity of other infiltration parameters decreases as the cumulative infiltration increases. At last, once intense rainfall stops, run-on produces infiltration mainly in the drainage network and progressively decreases.

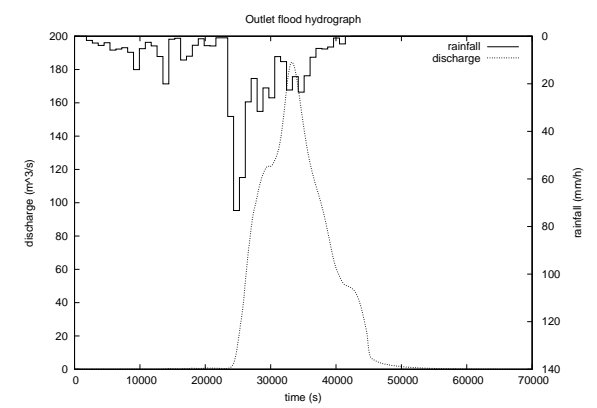

(a) Outlet hydrograph

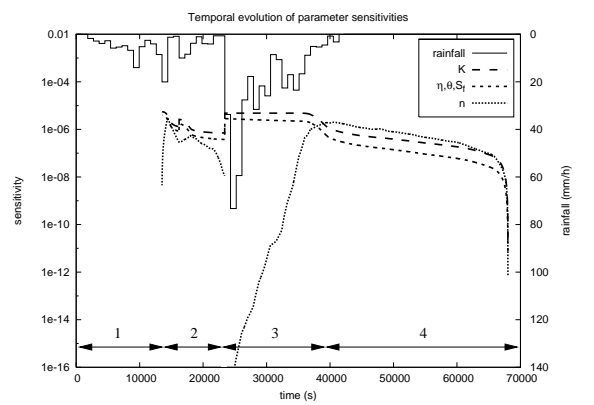

(b) Temporal patterns of sensitivities

Fig. 6. Sensitivity to parameters and flood hydrograph

For the considered test case, the potential of the adjoint sensitivity procedure was demonstrated for model diagnostic and sensitivity analysis. However, the behavior of the hydrosystem is analyzed only locally in the parameter space around effective values of model parameters and lead to sensitivities for a single point of the surface response. The influence of the point chosen for this local sensitivity analysis should be investigated in order to draw more general conclusions. Indeed, results may depend on the type of soil, on the ratio between rainfall intensity (also duration) and hydraulic conductivity. The contribution of some form of global sensitivity analysis should be examined. The development of automatic calibration methods received much attention from hydrologic community, but problems of differentiability, parameter insensitivity, parameters interactions and multiple local optima make the use of gradient based methods very difficult. However, even if the temporal increments of adjoint variables exhibit non-differentiable features, sensitivities 
of the global and smooth hydrological response to temporally integrated sensitivities should not. Systematic preliminary sensitivity analysis should also be carried out in order to identify the key parameters which really affect the chosen hydrological response. This should prevent from flat portions of surface response (i.e. low sensitivities for one direction of the parameter space). From the observed trends, a multi-criteria calibration strategy could be developed in order to adjust some of the parameters for a given aspect of the response. Such investigations are in progress and require appropriate regularization approaches and strategies for the reduction of the control space to be developed. Then, the well posed inverse problem can be solved using standard unconstrained optimization methods.

\section{Conclusion}

The adjoint method is a very efficient and flexible mathematical tool to calculate derivatives of a function of model state variable w.r.t. control variables. Two applications for flood modeling were presented in this prospective study. Variational data assimilation allows the identification of model parameters, initial and boundary conditions. Adjoint sensitivity analysis provides knowledge of the contribution of model inputs to the variations of some features of the solution, as well as a physical insight into the model dynamics.

The practical implementation of the adjoint method is significantly facilitated by the use of efficient AD tools like TAPENADE. The development time is considerably reduced and many human errors are avoided. However, with the current version of the tool, it is still necessary to manually modify the adjoint code to make it work properly. Unnecessary storage of variables can make the code require too much memory and the adjoint of some non-differentiable statements need to be manually fixed.

Nevertheless, AD remains a very powerful technique used for the achievement of the adjoint method. It opens new trends for the construction of an hydro-meteorological prediction chain and for future contributions concerning flood hazard forecast and mitigation.

\section{References}

1. F. Bouttier and P. Courtier. Data assimilation concepts and methods. Meteorological Training Course Lecture Series, ECMwF, 1999.

2. D.G. Cacuci. Sensitivity theory for nonlinear systems. I. Nonlinear functional analysis approach. J. Math. Phys., 22(12):2794-2802, 1981.

3. D.G. Cacuci. Sensitivity theory for nonlinear systems. II. Extensions to additional classes of responses. J. Math. Phys., 22(12):2803-2812, 1981.

4. D.G. Cacuci. Sensitivity and Uncertainty Analysis, Volume I Theory. Chapman \& Hall/CRC, 2003. 
5. F.-X. Le Dimet and O. Talagrand. Variational algorithms for analysis and assimilation of meteorogical observations. Tellus, 38A:97-110, 1986.

6. V. Estupina-Borrell, M.M. Maubourguet, J. Chorda, M. Alquier, and D. Dartus. Use of direct simulation whith space technology for flash flood events analysis. Ecosystems and Floods, Hanö̈, pages 72-87, 2000.

7. J.-C. Gilbert and C. Lemaréchal. Some numerical experiments with variable storage quasi-Newton algorithms. Mathematical programming, 45:407-435, 1989.

8. L. Hascoët, R.-M. Greborio, and V. Pascual. Computing adjoints by automatic differentiation with TAPENADE. In École INRIA-CEA-EDF "Problèmes nonlinéaires appliqués". Springer, 2003. To appear.

9. L. Hascoët, V. Pascual, and R.-M. Greborio. The TAPEnAde AD tool, Tropics Project, INRIA Sophia-Antipolis. AD Workshop, Cranfield, June 5-6, 2003.

10. J.-L. Lions. Optimal control of systems governed by partial differential equations. Springer-Verlag, 1971.

11. Y. Loukili and A. Soulaïmani. Numerical tracking of shallow water waves by the unstructured Finite Volume WAF approximation. Journal of Computational Methods in Sciences and Engineering, to appear, 2004.

12. C. Mazauric. Data assimilation for hydraulic models. Parameters estimation, sensitivity analysis and domain decomposition. $\mathrm{PhD}$ thesis, Université Joseph Fourier (Grenoble), 2003. In french.

13. P. Ngnepieba, F.-X. Le Dimet, A. Boukong, and G. Nguetseng. Inverse problem formulation for parameters determination using the adjoint method. ARIMA Journal - Revue Africaine de la Recherche in Informatique et Mathématiques Appliquées, 1:127-157, 2002.

14. InRIa, Tropics Project. TAPEnAde 2.0. <http://www-sop.inria.fr/tropics/>.

15. E.F. Toro. Shock-capturing methods for free-surface shallow flows. J. Wiley and Sons, Chichester, 2001.

16. P.A. Vidard, A. Piacentini, and F.-X. Le Dimet. Variational data analysis with control of the forecast bias. Tellus, 56A(3):177-188, 2004.

17. L.W. White, B. Vieux, D. Armand, and F.-X. Le Dimet. Estimation of optimal parameters for a surface hydrology model. Advances in Water Resources, 26(3):337-348, 2003.

18. J. Yang. Variational data assimilation for the problems of sediment transport in rivers. PhD thesis, Université Joseph Fourier (Grenoble), 1999. In french. 